\title{
Post-pandemic dentistry - restart or reform?
}

\author{
Psychological distress and the perceived impact of the COVID-19 pandemic on UK dentists during a national lockdown. \\ BrDent J 2021; 230: https://doi.org/10.1038/s41415-020-2592-5.
}

As dentists, we are well aware that our profession can be acutely stressful. We might expect that it has something to do with carrying out manually dextrous work at a ferocious pace, serenaded by high frequency handpieces under artificial light and the everpresent threat of litigation. Throw in a good measure of distrust from the public, together with dysfunctional systems of remuneration, then we might expect to feel occasionally

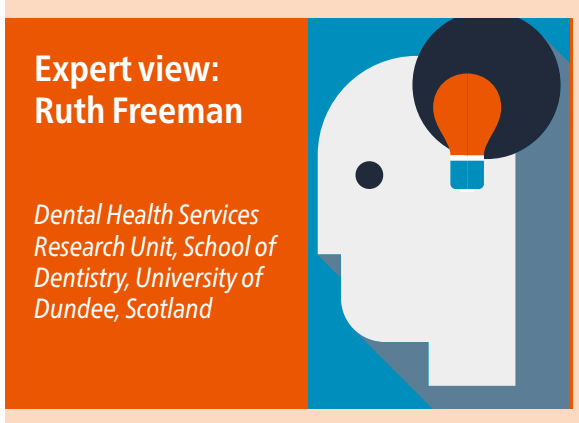

The emergence of COVID-19, in late December 2019 and the resulting pandemic, ${ }^{1}$ reflects Laurie Garrett's thesis in her book The coming plague, newly emerging diseases in a world out of balance. ${ }^{2}$ She proposes that it is the loss of habitat and global travel that provides an environment for novel infections to emerge, to be transmitted and for pandemics to occur. With people contracting the virus, becoming ill, experiencing morbidities and death, populations become overwhelmed by uncertainties and anxieties for their futures. It was into this mix, that the dental profession found itself in early 2020. Initial uncertainties of COVID-19 transmission, the role of AGPs, a scarcity of PPE, closed surgeries and anxieties regarding livelihoods, resulted in anecdotes of increased distress.

Therefore, this survey of $5,170 \mathrm{UK}$ dentists, during the third month of the first national lockdown, was timely. It showed that contrary to expectations dentists exhibited less distress. However, for those who were practice owners and whose NHS commitment was up to $49 \%$, they reported overwhelmed. What we might not have expected, however, is that a global pandemic could reduce distress.

The authors of this paper collected data from a survey of UK dentists in May 2020 and compared their findings with previous research. Their aim was to compare levels of psychological distress in UK dentists, before and during the COVID-19 pandemic. Just over 5,000 of our colleagues responded,

high levels of distress. For some dentists the release from practice was welcomed whereas for others, uncertainties of their financial situation, fears of bankruptcy and anxieties for family life were often mentioned. A deterioration of mental health functioning was noted. Distrust of regulatory bodies, public perceptions of the profession and worries of an uncertain future were commonplace. A call to support practitioners with 'reduced patient numbers but increased costs' and to monitor mental health status was made.

Regulatory and professional bodies must take heed of this call and devise support structures to enable the profession to continue to provide high quality care. Deaneries as part of their duty of care to those in training and in practice must construct interventions to monitor and promote mental health in all members of the dental team. The uncertainties and anxieties made manifest in the profession by the COVID-19 pandemic have critically exposed the difficulties experienced by the profession. It's time for action. The regulatory and professional bodies and the Deaneries must act now and support those who provide the oral healthcare for our population.

\section{References}

1. Liu YC, Kuo RL, Shin-Ru Shih SR. COVID-19: The first documented coronavirus pandemic in history, Biomed J 2020; 43: 328-333.

2. Garret L. The Coming Plague, Newly Emerging Diseases in a World Out of Balance. Harmonsworth: Penguin Books, 1996 accounting for roughly $12 \%$ of all UK dentists registered with the GDC. Overall, $57.8 \%$ of respondents exceeded the clinical threshold for psychological distress. Surprisingly, this represents a $10 \%$ decrease when compared to research carried out before the pandemic in 2017. Most responses were from GDPs, among whom practice owners reported significantly higher levels of psychological distress than associates. This could reasonably be predicted considering the additional financial burdens and responsibilities that come with running a business. The survey also gave respondents the opportunity to comment on how the pandemic has affected them and how they believe it will affect future practice. What emerges from these personal testimonies is an overwhelming sense of frustration. Colleagues describe themselves as 'completely disillusioned', feeling 'traumatised' and even being 'totally indifferent about the future of [their] career in dentistry'. There is deep dissatisfaction expressed towards those who are leading the profession with respondents often feeling undervalued and undersupported. However, many dentists described the time away from the profession - afforded by the pandemic - as beneficial. More time with family, indulging in hobbies and 'stepping off the treadmill' were all cited as positive changes brought about by the national lockdown.

Might we find some seeds of hope amidst these findings? Surely, the abrupt intrusion of COVID-19 has shaken the practice of dentistry in the UK like never before. How many of us have gained new perspective on life and work? As the engine of our profession shudders into action and gathers pace once more, how many of us will be prepared to sacrifice our newfound freedom and mental wellbeing? It seems that the national lockdown has given us all something we sorely needed - time. Time to relax, reflect and recharge. Surely now, we have the insight and motivation required to push for reform so that together with our colleagues, we may not merely function but thrive in our chosen profession.

Anne C. Devlin DCT3 OMFS, NHS Lothian 